\title{
Numerical Comparison between Different Lie-Group Methods for Solving Linear Oscillatory ODEs
}

\author{
Fasma Diele $^{1}$ and Stefania Ragni ${ }^{2}$ \\ 1 Istituto per Ricerche di Matematica Applicata, CNR, Bari, Italy \\ irmafd03@area.ba.cnr.it \\ 2 Facoltà di Economia, Università degli Studi di Bari, Bari, Italy \\ irmasr18@area.ba.cnr.it
}

\begin{abstract}
In this paper we deal with high oscillatory systems and numerical methods for the approximation of their solutions. Some classical schemes developed in the literature are recalled and a recent approach based on the expression of the oscillatory solution by means of the exponential map is considered. Moreover we introduce a new method based on the Cayley map and provide some numerical tests in order to compare the different approaches.
\end{abstract}

\section{Introduction}

Let us consider an initial-value ordinary differential system

$$
\mathbf{y}^{\prime}=\mathbf{f}(t, \mathbf{y}), \quad t \geq 0, \quad \mathbf{y}(0)=\mathbf{y}_{0}
$$

whose solution oscillates with a timescale much shorter than the integration interval. We will refer to these kind of dynamical systems as highly oscillatory ones. Indeed this concept is very generic; a more precise definition can be found in a survey provided by L. R. Petzold et al. in 1997 (see [10]) where it was stated that such equations are characterized by a fast solution varying regularly about a slow solution.

High oscillatory systems arise in many applications such as vehicle simulations, molecular dynamics, circuit simulations, flexible body dynamics. In order to provide some examples of oscillatory differential problems, let us consider the solution of

$$
y^{\prime \prime}+t y=0, \quad t \geq 0,
$$

given by $y(t)=\pi\left[A i(-t) B i^{\prime}(0)-A i^{\prime}(0) B i(-t)\right]$, which satisfies the initial conditions $y(0)=1, y^{\prime}(0)=0$, where $A i(z)$ is the so-called Airy function and Bi(z) represents the Airy function of the second kind. As another example, the Bessel function of index $\nu=0$

$$
J_{0}(t)=\sum_{k=0}^{\infty} \frac{(-1)^{k}}{(k !)^{2}}\left(\frac{t}{2}\right)^{2 k}
$$


is the solution of the following equation

$$
t^{2} y^{\prime \prime}+t y^{\prime}+t^{2} y=0, \quad t \geq 0 .
$$

In Figure 1 we plot the Airy function behaviour and the approximation of (2) obtained by means of the Besselj built-in Matlab code.
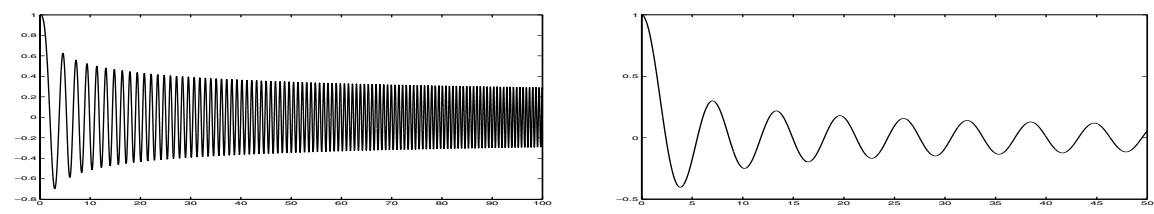

Fig. 1. Plot of the Airy (left) and the Bessel (right) functions.

Different approaches for the numerical solution of this kind of problems have been developed in the literature. With the aim to investigate the stability features of the classical numerical methods, such as multistep and Runge-Kutta ones, applied to oscillatory systems, the scalar harmonic oscillator equation

$$
y^{\prime \prime}=-\omega^{2} y, \quad \omega>0
$$

is usually chosen as standard test problem. In this respect, a suitable stability definition is due to Lambert and Watson who originally introduced the concept of $P$-stability (see [9]). We notice that, since the step length is constrained not only by stability but also by accuracy, then a stepsize of the same magnitude as the period of oscillation with highest frequency is required even for $P$-stable methods. The form and structure of the effective numerical methods is strongly application-dependent, so they vary according to specific classes of the considered applications. For instance, when the presence of forcing terms arises in some applications, it is interesting to account for the following inhomogeneous equation:

$$
\mathbf{y}^{\prime \prime}=A(t) \mathbf{y}(t)+\mathbf{b}(\mathbf{y}) .
$$

In the case when $A(t)=A$, the solution can be obtained by means of the mollified impulse method due to Garcia-Archilla, Sanz-Serna and Skeel [2]. Successively in [3] Hochbruch and Lubich introduced the Gautschi-type methods that reduce to solve exactly the equation with constant inhomogeneity.

Recently Iserles proposed a completely different approach based on the Magnus expansion (see [5]). More precisely the solution of the linear system $\mathbf{y}^{\prime}=$ $A(t) \mathbf{y}, \mathbf{y}(0)=\mathbf{y}_{0}$ is represented in terms of the exponential matrix $\mathbf{y}(t)=e^{\Omega(t)} \mathbf{y}_{0}$ where $\Omega(t)$ satisfies a suitable differential equation, as we recall in Section 2. By following this idea we propose to express the same solution in terms of the Cayley transform $\mathbf{y}(t)=\left(I-\frac{1}{2} \Omega(t)\right)^{-1}\left(I+\frac{1}{2} \Omega(t)\right) \mathbf{y}_{0}$ with $\Omega(t)$ satisfying a related equation, as shown in Section 3. We notice that these approaches arise in a completely different field such as the numerical approximation of conservative 
differential problems (see e.g. [7]). The idea to apply the Cayley transform in the framework of oscillatory systems is suggested by its competitiveness and cheapness with respect to the exponential map. In Section 4 we compare these two approaches verifying their effectiveness on the Airy and Bessel test problems. Finally, conclusion and suggestions for further research are discussed in Section 5 .

\section{The Magnus methods}

In this section we sketch some basic idea about methods based on the Magnus expansion adopting the same notations used in [7]. It is known that the solution of

$$
\mathbf{y}^{\prime}=A(t) \mathbf{y}(t) \quad t \geq 0, \quad \mathbf{y}(0)=\mathbf{y}_{0}
$$

can be written in the form

$$
\mathbf{y}(t)=e^{\Omega(t)} \mathbf{y}_{0}
$$

where $\Omega$ satisfies the following equation

$$
\Omega^{\prime}=\sum_{k=0}^{\infty} \frac{B_{k}}{k !} \operatorname{ad}_{\Omega}^{k} A, \quad t \geq t_{0}, \quad \Omega\left(t_{0}\right)=O
$$

being $\left\{B_{k}\right\}_{k \in \mathbb{Z}^{+}}$the Bernoulli numbers and

$$
\begin{aligned}
\operatorname{ad}_{\Omega}^{0} A & =\Omega \\
\operatorname{ad}_{\Omega}^{k} A & =\left[A, \operatorname{ad}_{\Omega}^{k-1} A\right]=A \operatorname{ad}_{\Omega}^{k-1} A-\operatorname{ad}_{\Omega}^{k-1} A A, \quad k>0 .
\end{aligned}
$$

The solution of the previous system is the so-called Magnus expansion of $\Omega$ given by

$$
\begin{aligned}
& \Omega(t)=\int_{0}^{t} A(\xi) d \xi-\frac{1}{2} \int_{0}^{t} \int_{0}^{\xi_{1}}\left[A\left(\xi_{2}\right), A\left(\xi_{1}\right)\right] d \xi_{2} d \xi_{1} \\
& +\frac{1}{12} \int_{0}^{t} \int_{0}^{\xi_{1}} \int_{0}^{\xi_{1}}\left[A\left(\xi_{3}\right),\left[A\left(\xi_{2}\right), A\left(\xi_{1}\right)\right]\right] d \xi_{3} d \xi_{2} d \xi_{1} \\
& +\frac{1}{4} \int_{0}^{t} \int_{0}^{\xi_{1}} \int_{0}^{\xi_{2}}\left[\left[A\left(\xi_{3}\right), A\left(\xi_{2}\right)\right], A\left(\xi_{1}\right)\right] d \xi_{3} d \xi_{2} d \xi_{1}+\ldots
\end{aligned}
$$

In order to discretize the solution (5), it is necessary to truncate the infinite Magnus expansion (6) and to replace integrals by quadrature. Therefore the Magnus numerical scheme consists of advancing the Magnus expansion by step $h>0$ and approximating $\mathbf{y}\left(t_{n+1}\right)=e^{\Omega_{n}(h)} \mathbf{y}\left(t_{n}\right)$, by

$$
\mathbf{y}_{n+1}=e^{\tilde{\Omega}_{n}(h)} \mathbf{y}_{n}
$$

with $\tilde{\Omega}_{n}(h)$ truncation of $\Omega_{n}(h)$, where the integrals are replaced by quadrature. 


\subsection{Modified Magnus methods}

A modified version of the Magnus method can be designed explicitly for oscillatory systems as in [5]. The algorithm advances from $t_{n}$ to $t_{n}+h$ by setting

$$
\mathbf{y}(t)=\mathbf{e}^{\left(t-t_{n}\right) A\left(t_{n+1 / 2}\right)} \mathbf{x}(t), \quad t \geq t_{n}
$$

where $t_{n+1 / 2}=t_{n}+\frac{1}{2} h$ and the function $\mathbf{x}(t)$ satisfies

$$
\mathbf{x}^{\prime}=B(t) \mathbf{x}, \quad t \geq t_{n}, \quad \mathbf{x}\left(t_{n}\right)=\mathbf{y}\left(t_{n}\right)
$$

with $B(t)=\mathbf{e}^{\left(t-t_{n}\right) A\left(t_{n+1 / 2}\right)}\left[A(t)-A\left(t_{n+1 / 2}\right)\right] \mathbf{e}^{\left(t-t_{n}\right) A\left(t_{n+1 / 2}\right)}$. The latter equation is discretized by the standard Magnus method so that $\mathbf{x}(t)=\mathbf{e}^{\tilde{\Omega}_{n}(t)} \mathbf{y}_{n}$; therefore the global approximation is given by

$$
\mathbf{y}_{n+1}=\mathbf{e}^{h A\left(t_{n+1 / 2}\right)} \mathbf{e}^{\tilde{\Omega}_{n}(h)} \mathbf{y}_{n}, \quad n \in \mathbb{Z}_{+} .
$$

Remark 1. The idea which is the basis of this algorithm, is that the oscillatory behaviour of (4) is locally well modelled by the linear equation with constant coefficients

$$
\tilde{\mathbf{y}}^{\prime}=A\left(t_{n+1 / 2}\right) \tilde{\mathbf{y}}
$$

whose solution is given by a matrix exponential.

\section{The Cayley method}

Following the same idea developed by Iserles, we apply a method based on the Cayley map in order to solve an oscillatory system. As a counter part of (5), the solution of (4) is given by

$$
\mathbf{y}(t)=\operatorname{cay}(\Omega(t)) \mathbf{y}_{0}
$$

where cay $(\Omega(t))=\left[I-\frac{1}{2} \Omega(t)\right]^{-1}\left[I+\frac{1}{2} \Omega(t)\right]$ is the Cayley transform of the matrix $\Omega(t)$ that satisfies the following equation (see [1], [4])

$$
\Omega^{\prime}=A-\frac{1}{2}[\Omega, A]-\frac{1}{4} \Omega A \Omega, \quad t \geq t_{0}, \quad \Omega\left(t_{0}\right)=O .
$$

Notice that evaluating the Cayley transform is cheaper than computing a matrix function exponential. It is possible to solve (8) by performing the Magnus expansion of $\Omega$ (see [4])

$$
\begin{aligned}
& \Omega(t)=\int_{0}^{t} A(\xi) d \xi-\frac{1}{2} \int_{0}^{t} \int_{0}^{\xi_{1}}\left[A\left(\xi_{2}\right), A\left(\xi_{1}\right)\right] d \xi_{2} d \xi_{1} \\
& -\frac{1}{4} \int_{0}^{t}\left[\int_{0}^{\xi_{1}} A\left(\xi_{2}\right) d \xi_{2}\right] A\left(\xi_{1}\right)\left[\int_{0}^{\xi_{1}} A\left(\xi_{3}\right) d \xi_{3}\right] d \xi_{1} \\
& +\frac{1}{4} \int_{0}^{t} \int_{0}^{\xi_{1}} \int_{0}^{\xi_{2}}\left[\left[A\left(\xi_{3}\right), A\left(\xi_{2}\right)\right], A\left(\xi_{1}\right)\right] d \xi_{3} d \xi_{2} d \xi_{1}+\ldots
\end{aligned}
$$


Again, we can achieve the numerical solution by truncating the infinite Magnus expansion (9) and replacing integrals by quadrature. Therefore the Cayley numerical scheme is

$$
\mathbf{y}_{n+1}=\operatorname{cay}\left(\tilde{\Omega}_{n}(h)\right) \mathbf{y}_{n}
$$

with $h>0$ and $\tilde{\Omega}_{n}(h)$ the truncation of $\Omega_{n}(h)$ obtained replacing the integrals by quadrature.

\subsection{Modified Cayley method}

As a counterpart of the modified Magnus method, we consider again the algorithm that advances from $t_{n}$ to $t_{n}+h$ by defining

$$
\mathbf{y}(t)=\mathbf{e}^{\left(t-t_{n}\right) A\left(t_{n+1 / 2}\right)} \mathbf{x}(t), \quad t \geq t_{n}
$$

where $\mathbf{x}(t)$ satisfies (7). In this case, we discretize equation (7) using the standard Cayley method; therefore we obtain the solution

$$
\mathbf{y}_{n+1}=\mathbf{e}^{h A\left(t_{n+1 / 2}\right)} \operatorname{cay}\left(\tilde{\Omega}_{n}(h)\right) \mathbf{y}_{n}, \quad n \in \mathbb{Z}_{+} .
$$

\section{Numerical schemes and results}

We are going to list the methods described so far. More precisely, we consider the fourth order schemes for Magnus and Cayley methods already presented in [7]. In the sequel we will denote for each $n$

$$
A_{i}=A\left(t_{n}+c_{i} h\right) \quad i=1,2
$$

with $c_{1}=\frac{1}{2}-\frac{\sqrt{3}}{6}, c_{2}=\frac{1}{2}+\frac{\sqrt{3}}{6}$. Concerning the fourth order Magnus scheme (Magnus4), we compute $y_{n+1}=\exp \left(\Omega_{n}\right) y_{n}$ where $\Omega_{n}$ is given by

$$
\Omega_{n}=\frac{h}{2}\left(A_{1}+A_{2}\right)-\frac{\sqrt{3}}{12} h^{2}\left[A_{1}, A_{2}\right] .
$$

As a counterpart, the fourth order method based on the Cayley expansion (Cayley4) provides the approximation $y_{n+1}=\operatorname{cay}\left(\Omega_{n}\right) y_{n}$ by evaluating

$$
\Omega_{n}=h B_{0}+\frac{1}{12} h^{2}\left[B_{1}, B_{0}\right]-\frac{1}{12} h^{3} B_{0}^{3}
$$

where $B_{0}=\frac{1}{2}\left(A_{1}+A_{2}\right)$ and $B_{1}=\sqrt{3}\left(A_{2}-A_{1}\right)$. We implement the modified versions of the previous methods by following the same schemes given in [5].

Our aim is to validate the effectiveness of the Cayley approach in the field of oscillatory systems by applying it on the Airy and Bessel equations which we consider as test problems. Moreover, we are interested in the comparison between the different schemes based on the exponential and the Cayley maps. In each figure we plot, on different timescales, the errors in the solution of the test problems (1) and (3) by using Magnus and Cayley approaches with time 

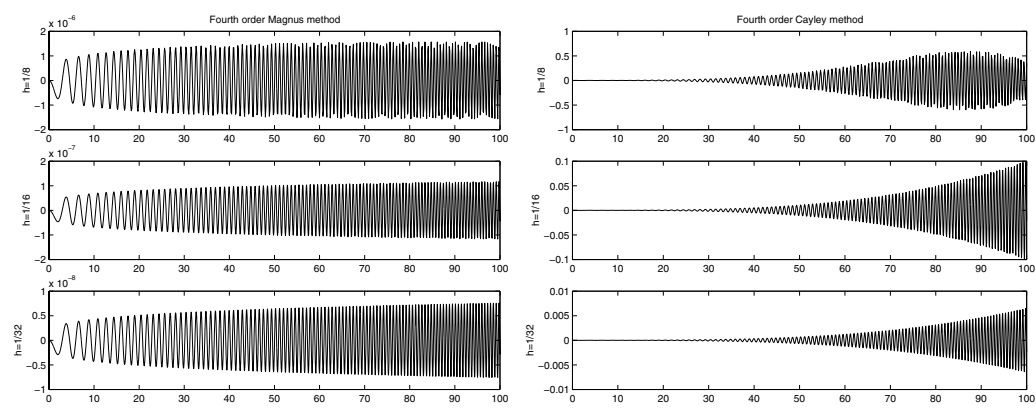

Fig. 2. The error in the solution of the Airy equation (1) by Magnus4 (left) and Cayley4 (right) in the time interval $[0,100]$.
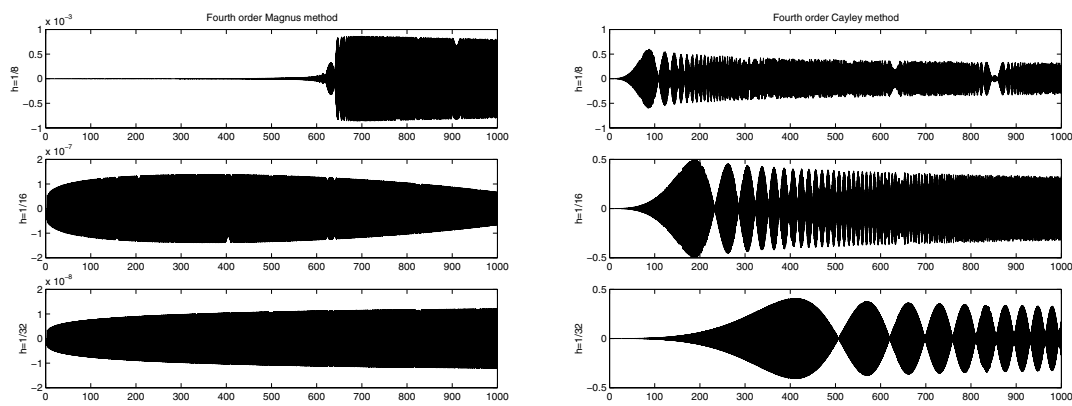

Fig. 3. The error in the solution of the Airy equation (1) by Magnus4 (left) and Cayley4 (right) in the time interval $[0,1000]$.

steps $h=\frac{1}{8}, h=\frac{1}{16}$ and $h=\frac{1}{32}$. In Figures 2 and 3 , it is evident that the Magnus method performs better than the Cayley one on both timescales, when applied to the Airy equation; anyway, notice that it loses accuracy for the longer integration interval. Concerning the modified versions, as shown in Figures 4 and 5, both the approaches behave similarly. However, the Cayley approach takes a sharp improvement when implemented in modified version. Otherwise, this does not hold for the Magnus schemes. We notice that in [5] and in [6] a remarkable advantage is get out of the modified approach performing the Magnus expansion with exact integrals. We can make similar considerations regarding the performances of the considered schemes for the solution of the Bessel equation. Even if the results in Figures 6 and 7 obtained by applying Cayley4 are acceptable, they are not competitive with respect to the Magnus4 ones. Again, as shown in Figures 8 and 9, a clear improvement is achieved when the modified version of the Cayley method is used. 

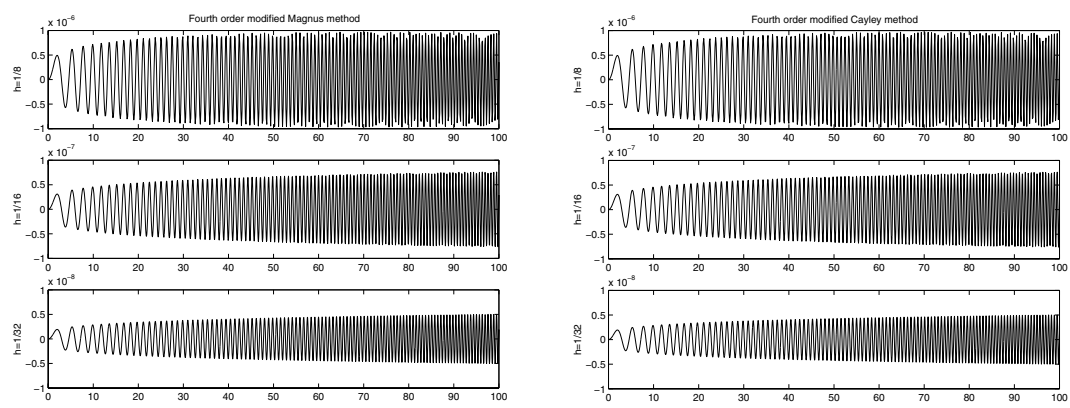

Fig. 4. The error in the solution of the Airy equation (1) by modified Magnus4 (left) and modified Cayley 4 (right) in the time interval $[0,100]$.
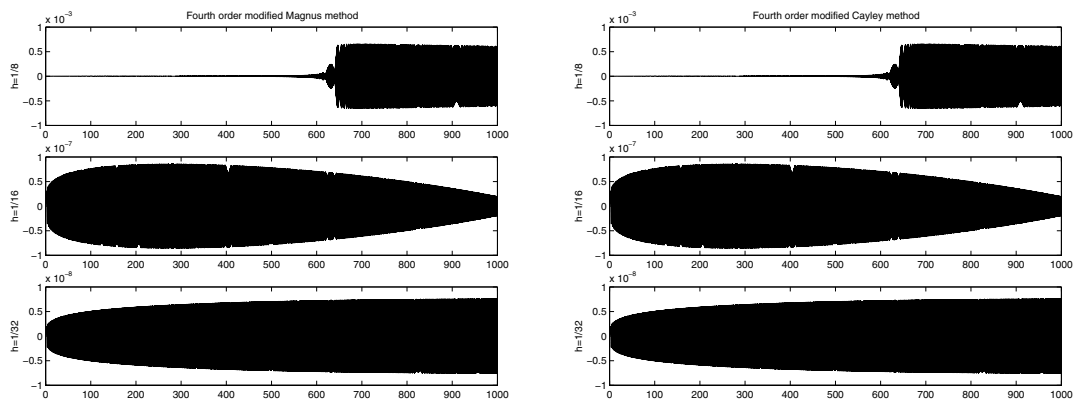

Fig. 5. The error in the solution of the Airy equation (1) by modified Magnus4 (left) and modified Cayley4 (right) in the time interval [0, 1000].
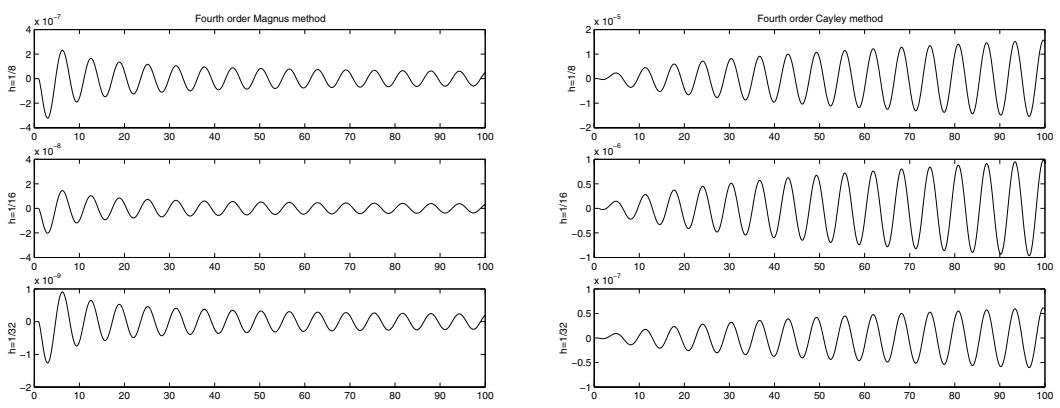

Fig. 6. The error in the solution of the Bessel equation (3) by Magnus4 (left) and Cayley4 (right) in the time interval $[1,100]$. 

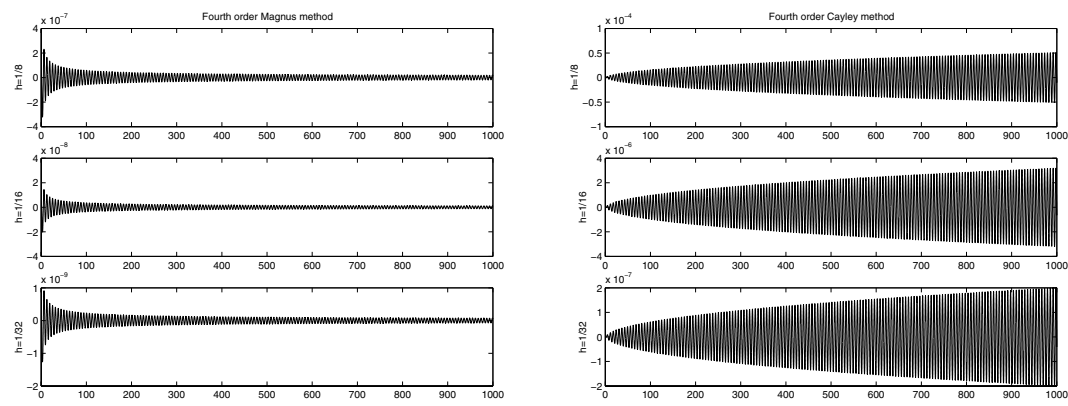

Fig. 7. The error in the solution of the Bessel equation (3) by Magnus4 (left) and Cayley4 (right) in the time interval $[1,1000]$.
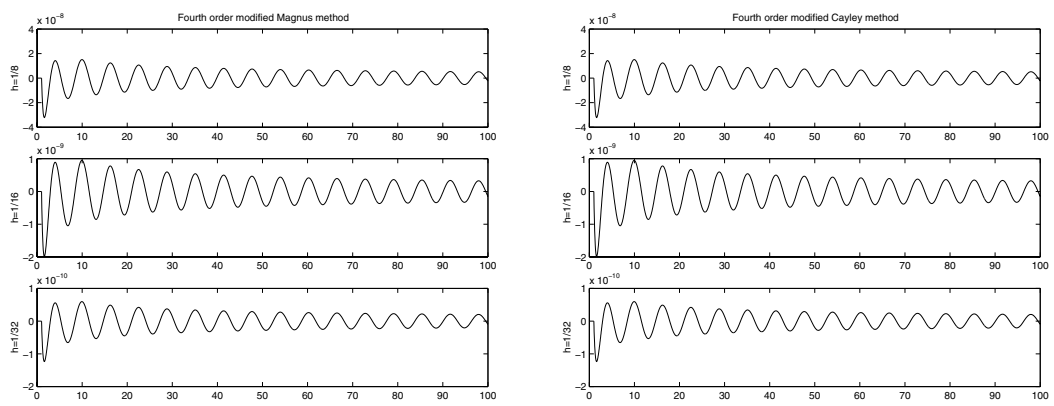

Fig. 8. The error in the solution of the Bessel equation (3) by modified Magnus4 (left) and modified Cayley4 (right) in the time interval $[1,100]$.
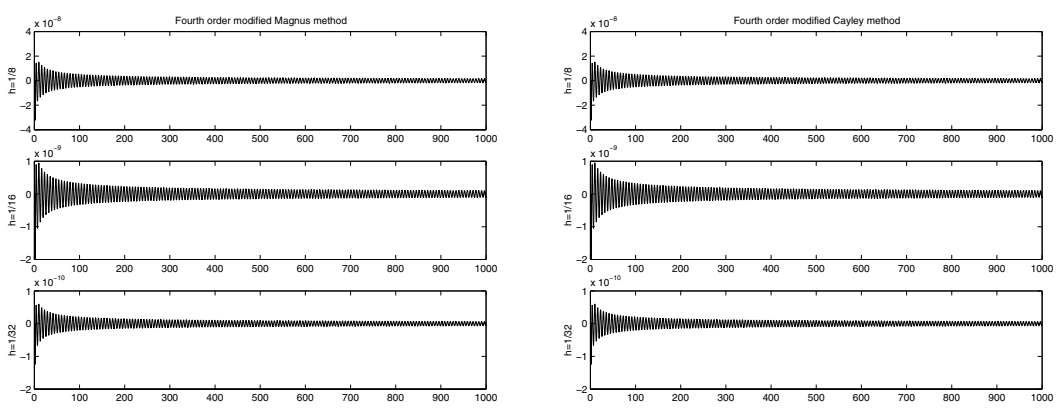

Fig. 9. The error in the solution of the Bessel equation (3) by modified Magnus4 (left) and modified Cayley4 (right) in the time interval $[1,1000]$. 


\section{Conclusions and further research}

To solve highly oscillatory differential systems, we proposed a numerical approach which is based on the solution expression by means of the Cayley transform. We performed several numerical tests in order to show the effectiveness of this approach with respect to the Magnus methods.

A modified version of the considered methods, explicitly designed for oscillatory problems, is taken into account. We point out the cheapness and good performance of the modified scheme based on the Cayley transform with respect to ones based on the exponential map.

Further research will intend to provide theoretical results which justify the good performance of the proposed method. Moreover, we will extend the present approach to the non homogeneous case. It might be advantageous to use the Cayley method for the oscillatory part and following an approach like Gautschitype to treat the non oscillatory term.

\section{References}

1. Diele, F., Lopez, L., Peluso, R.: The Cayley transform in the numerical solution of unitary differential systems. Adv. Comput. Math. 8 (1998) 317-334

2. Garca-Arcilla, B., Sanz-Serna, J. M., Skeel, R. D.: Long-time-step methods for oscillatory differential equations. SIAM J. Sci. Comput. 20 No.3 (1998) 930-963

3. Hochbruck, M., Lubich, C.: A Gautschi-type method for oscillatory second-order differential equations. Numer. Math. 83 (1999) 403-426

4. Iserles, A.: On Cayley-transform methods for the discretization of Lie-group equations. Technical report 1999/NA04. Department of Applied Mathematics and Theoretical Physics, University of Cambridge.

5. Iserles, A.: On the global error of discretization methods for highly-oscillatory ordinary differential equations. Technical report 2000/NA11. Department of Applied Mathematics and Theoretical Physics, University of Cambridge.

6. Iserles, A.: Think globally, act locally: solving highly-oscillatory ordinary differential equations. Technical report 2001/NA06. Department of Applied Mathematics and Theoretical Physics, University of Cambridge.

7. Iserles, A., Munthe Kaas, H. Z., Norsett, S. P., Zanna, A.: Lie-groups methods. Acta Numerica 9 (2000) 215-365

8. Iserles, A., Norsett, S. P., Rasmussen, A. F.: Reversibility and high-order Magnus methods. Technical Report 1998/NA06. Department of Applied Mathematics and Theoretical Physics, University of Cambridge.

9. Lambert, J. D., Watson, I. A.: Symmetric multistep methods for periodic initial value problems. J. Inst. Math. Appl. 18 (1976) 189-202

10. Petzold, L. R., Jay, L. O., Yen, J.: Numerical solution of highly oscillatory ordinary differential equations. Acta Numerica 6 (1997) 437-483

\section{Appendix: MATLAB code}

For the sake of completeness, herewith a MATLAB code for the fourth order modified Cayley method: 
function $[t, Y]=\operatorname{MCayley} 4(\mathrm{~N}, \mathrm{to}, \mathrm{tN}, \mathrm{y} 0, \mathrm{~h})$;

$\%$ Modified Cayley4

$\%$ Input:

$\% \mathrm{~N}$ : the spacing of solution points (typically $\mathrm{N}=1$ )

$\%$ to: initial point

$\%$ tN: endpoint

$\%$ y0: initial value

$\%$ h: step size

$\%$ Output:

$\% \quad \mathrm{t}=[\mathrm{t0}: \mathrm{Nh}: \mathrm{tN}]$

$\% \quad Y=[y(t 0), y(t 0+N h), y(t 0+2 N h), \ldots, y(t N)]$

$\mathrm{c} 1=(1 / 2-\operatorname{sqrt}(3) / 6) * \mathrm{~h}$;

$c 2=(1 / 2+\operatorname{sqrt}(3) / 6) * h$;

$\mathrm{h} 2=\mathrm{h} / 2$;

$\mathrm{t}=\mathrm{t} 0$;

$\mathrm{Y}=\mathrm{y} 0$

$I=\operatorname{eye}($ length $(\mathrm{y} 0)) ;$

$M=f l o o r((t N-t 0) / h+e p s) ;$

for $\mathrm{n}=1: \mathrm{M}$

$\mathrm{AO}=\operatorname{Afun}(\mathrm{tO}+\mathrm{h} 2) ;$

$\mathrm{Th}=\operatorname{expm}(\mathrm{h} * \mathrm{AO})$;

$\mathrm{T} 1=\operatorname{expm}(\mathrm{c} 1 * \mathrm{~A} 0) ;$

$\mathrm{T} 2=\operatorname{expm}(\mathrm{c} 2 * \mathrm{~A} 0)$;

$A 1=\operatorname{Afun}(t 0+c 1) ;$

$A 2=\operatorname{Afun}(t 0+c 2)$;

$\mathrm{U} 1=\mathrm{T} 1 \backslash(\mathrm{A} 1-\mathrm{A} 0) * \mathrm{~T} 1$;

$\mathrm{U} 2=\mathrm{T} 2 \backslash(\mathrm{A} 2-\mathrm{A} 0) * \mathrm{~T} 2$;

$\mathrm{BO}=1 / 2 *(\mathrm{U} 1+\mathrm{U} 2)$;

$\mathrm{B} 1=\operatorname{sqrt}(3) *(\mathrm{U} 2-\mathrm{U} 1)$;

$\mathrm{C}=\mathrm{B} 1 * \mathrm{~B} 0-\mathrm{B} 0 * \mathrm{~B} 1$;

Theta $=\mathrm{h} * \mathrm{~B} 0+\mathrm{h} \bumpeq 2 / 12 * \mathrm{C}-1 / 12 * \mathrm{~h} \bumpeq 3 * \mathrm{~B} 0$ ^3;

$I 1=(I-1 / 2 *$ Theta $)$;

$I 2=(I+1 / 2 *$ Theta $)$;

$\mathrm{U}=\mathrm{I} 1 \backslash \mathrm{I} 2$;

$\mathrm{y} 0=\mathrm{Th} * \mathrm{U} * \mathrm{y} 0 ;$

$\mathrm{to}=\mathrm{to}+\mathrm{h}$;

if $f \operatorname{loor}(\mathrm{n} / \mathrm{N}) * \mathrm{~N}==\mathrm{n}$

$\mathrm{Y}=[\mathrm{Y}, \mathrm{yO}]$;

$t=[t, t 0]$;

end

end

where the function Afun.m provides the values of the matrix $A(t)$ : for instance, for the Airy equation (1) it reads

function $A=\operatorname{Afun}(t) A=[0,1 ;-t, 0]$ 